\title{
Modelling and Simulation of the Oxidative Stabilization Process of Pitch-based Carbon Fiber
}

\author{
Toshikuni Yonemoto, Hiroaki Nitta and Teiriki Tadaki \\ (Received December 24, 1991) \\ Department of Biochemistry and Engineering \\ Faculty of Engineering, Tohoku University \\ Aoba-ku, Sendai, Miyagi 980, Japan
}

\begin{abstract}
Oxidative stabilization of petroleum derived mesophase pitch fiber under increasing temperature conditions was simulated through the use of a kinetic model in which the mass transfer of the oxygen molecules and the autoxidation reaction with the active site in the fiber were taken into account. Temperature dependence of physicochemical parameters such as the effective diffusivity of oxygen molecules in the fiber, the reaction rate constant and the gas-solid equilibrium coefficient of oxygen molecules, were introduced into the mathematical model. The validity of the kinetic model and the numerical calculations was ascertained by comparison of the theoretical values for the outlet concentration of oxygen gas and the rate of oxygen consumption by the fibers with experimental values. The stabilization reaction was completed prior to the entire consumption of active sites in the fiber, and fixed oxygen was considered to be non-uniformly distributed in the direction of the fiber radius.
\end{abstract}

KEYWORDS: Oxidative stabilization, Pitch fiber, Kinetic model, Numerical simulation

\section{Introduction}

It is well known that the pitch-based carbon fiber developed by Otani ${ }^{1,2)}$ has future potential because of the low cost of source materials and excellent performance properties of the product. In the fabrication of the product, oxidative stabilization of pitch fiber is an indispensable step to hold the oriented structure of the fiber during subsequent carbonization. This step is also known to be the most time-consuming in the entire production process ${ }^{3)}$. Thus, it is important to elucidate the reaction mechanism of the stabilization and to explore the optimum operating conditions.

Recently, many researchers have conducted ${ }^{4)-8)}$ experimental studies on the subjects mentioned above and it is recognized that the oxidative stabilization is a very complicated rate process in which diffusion and reaction processes of oxygen molecules are coupled with each other. We also conducted the oxidative stabilization experiments of petroleum derived mesophase pitch fiber under several isothermal conditions $^{9)}$ and have proposed a kinetic model for isothermal processes ${ }^{10)}$.

In this paper, the previously proposed kinetic model of the oxidative stabilization is extended to apply to non-isothermal surroundings. Several experiments under increasing temperature conditions are simulated numerically with the new model equations.

\section{Experiment}

\subsection{Experimental apparatus and procedure}

The stabilization experiments under increasing temperature conditions were conducted in a gas-phase, perfectly-mixed reactor made of stainless steel with a stainless steel basket to hold the fibers. Figure 1 shows a detailed illustration of the reactor. The reactor was almost identical to that of the previous studies ${ }^{9)-11)}$ but the volume $\left(=984 \mathrm{~cm}^{3}\right)$ was less than that of the 


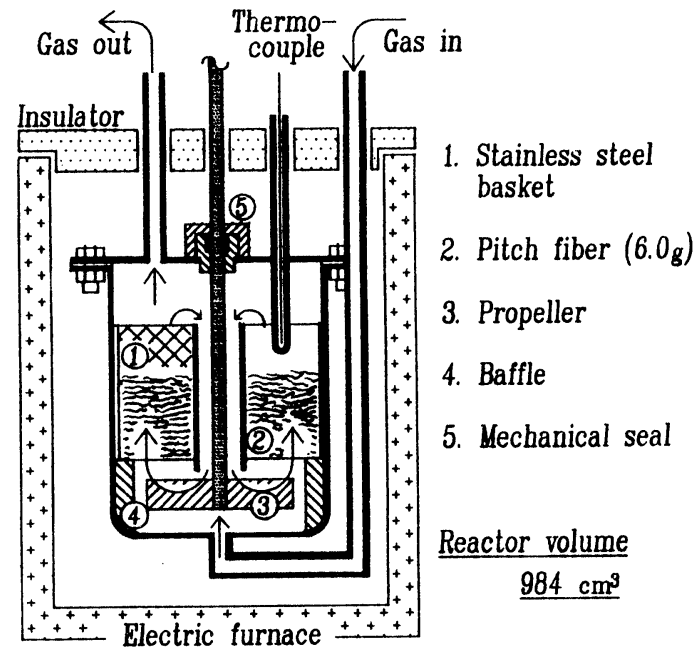

Fig. 1 Detail of reactor

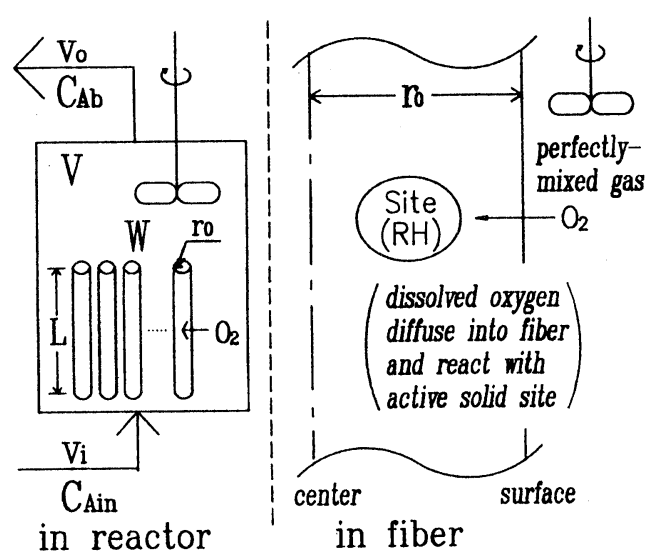

Fig. 2 Oxidative stabilization model

previous one.

Mixed reaction gas of $20 \mathrm{~mol} \% \mathrm{O}_{2}$ and $80 \mathrm{~mol} \%$ $\mathrm{N}_{2}$ supplied from cylinders was introduced through the tube connected to the reactor bottom. The gas-flow rate was regulated at $100 \mathrm{~cm}^{3} \cdot \mathrm{min}^{-1}$ [NTP]. Six grams of pitch fiber were packed into the basket in the reactor. The temperature in the reactor was raised at 2 or $4^{\circ} \mathrm{C} \cdot \mathrm{min}^{-1}$ heating to $240^{\circ} \mathrm{C}$ and then kept there by using a microcomputer and electric furnace. The exit gas was withdrawn at fixed time intervals by a gas sampler and analyzed for oxygen concentration by means of a gas chromatograph (Hitachi, 236-70-TCD).
Oxidized fiber removed from the reactor was then carbonized by burner heat to confirm the completion of the stabilization.

The stabilization experiments under several isothermal conditions ${ }^{9)}$ were also conducted to obtain temperature dependence of rate and equilibrium parameters ${ }^{10)}$.

\subsection{Sample fiber}

The pitch fibers used in the experiments originated from petroleum derived mesophase pitch. The diameter of the fiber was $1.5 \times 10^{-5} \mathrm{~m}(15 \mu \mathrm{m})$. Characteristics of the fiber were almost identical to those in the previous studies ${ }^{9), 10)}$ and the concentration of active sites estimated from the aliphatic carbon content in the fiber was $1.10 \times 10^{-2} \mathrm{~mol} \cdot \mathrm{cm}^{-3}$.

\section{Mathematical Modelling and Simulation}

Consider the gas-phase, perfectly-mixed reactor of volume $\mathrm{V}$, in which many pitch fibers of radius $r_{0}$ and total weight W, are packed as shown in Fig. 2. The temperature of the reactor is increased from absolute room temperature, $T_{R}$, to the prescribed maximum temperature, $T_{\max }$, at a constant rate, $a$, as follows:

$$
\begin{aligned}
& \mathrm{T}=\mathrm{T}_{\mathrm{R}}+\mathrm{a} \cdot \mathrm{t}\left(0 \leqq \mathrm{t}<\left(\mathrm{T}_{\max }-\mathrm{T}_{\mathrm{R}}\right) / \mathrm{a}\right) \\
& \mathrm{T}=\mathrm{T}_{\max } \quad\left(\mathrm{t} \geqq\left(\mathrm{T}_{\max }-\mathrm{T}_{\mathrm{R}}\right) / \mathrm{a}\right)
\end{aligned}
$$

Feed gas of flow rate $\mathrm{v}_{0}$ and oxygen concentration $\mathrm{C}_{\mathrm{A} 0}$ at room temperature is introduced into the reactor. The oxygen concentration in the reactor inlet, whose temperature is $T$, is expressed as follows:

$$
\mathrm{C}_{\text {Ain }}=\frac{\mathrm{T}_{\mathrm{R}}}{\mathrm{T}} \mathrm{C}_{\mathrm{A} 0}
$$

where $T_{R}$ is room temperature in Kelvin units.

Dissolved oxygen molecules diffuse toward the interior of the fiber and are consumed by the reaction with the active sites in the fiber according to the following rate equation ${ }^{10)}$ :

$$
-\mathrm{r}_{\mathrm{A}}=\mathrm{kC}_{\mathrm{s}} \mathrm{C}_{\mathrm{A}}
$$

where $\mathrm{k}$ is the reaction rate constant and $\mathrm{C}_{\mathrm{s}}$ is the concentration of the active sites, which is initially distributed uniformly in the pitch fiber.

The time variation in local oxygen and local active site concentrations can be written as ${ }^{10}$

$$
\frac{\partial C_{A}}{\partial t}=\frac{D_{E}}{r} \cdot \frac{\partial}{\partial r}\left(r \frac{\partial C_{A}}{\partial r}\right)-k C_{A} \cdot C_{S}
$$


and

$$
\frac{\partial \mathrm{C}_{\mathrm{S}}}{\partial \mathrm{t}}=-\mathrm{kC}_{\mathrm{A}} \cdot \mathrm{C}_{\mathrm{S}}
$$

where $D_{E}$ is the effective diffusivity of oxygen molecules in the fiber.

The gas-phase oxygen concentration in the reactor, $\mathrm{C}_{\mathrm{Ab}}$, can be formulated by using the mass balance equation as follows:

$$
\begin{aligned}
(1 & \left.-\frac{R T}{P} C_{A b}\right)\left.2 \pi r_{0} L \cdot D_{E} \frac{\partial C_{A}}{\partial r}\right|_{r}=r_{0} \\
= & v_{0}\left(C_{A i n}-C_{A b}\right)-V \frac{\partial C_{A b}}{\partial t}-\frac{V \cdot C_{A b}}{T} \cdot \frac{\partial T}{\partial t}
\end{aligned}
$$

where $\mathrm{L}\left(\equiv \rho \mathrm{W} /\left(\pi \mathrm{r}_{0}^{2)}\right)\right.$ is total fiber length. At the beginning of the reaction, the concentration of oxygen in the fibers is zero. Therefore, the initial conditions are established as follows:

$$
\mathrm{t}=0 ; \mathrm{C}_{\mathrm{A}}=0, \mathrm{C}_{\mathrm{S}}=\mathrm{C}_{\mathrm{S} 0}, \mathrm{C}_{\mathrm{Ab}}=\mathrm{C}_{\mathrm{A} 0}
$$

where $\mathrm{C}_{\mathrm{SO}}$ is the initial concentration of the active sites in the fiber.

Under the assumption of a linear gas-solid equilibrium at the fiber surface and no mass flux at the center of the fiber for oxygen molecules, the boundary conditions are given as follows:

$$
\begin{aligned}
& r=r_{0} ; C_{A}=H \cdot C_{A b} \\
& r=0 ; \frac{\partial C_{A}}{\partial r}=0
\end{aligned}
$$

where $\mathrm{H}$ is the gas-solid equilibrium constant.

The model equations, (1) to (9), are coupled partial differential equations and are firstly converted into the dimensionless forms and then rewritten in finite difference forms by means of the fully implicit CrankNicolson method. The process mentioned above is almost identical to that of our previous studies ${ }^{10), 11)}$ for an isothermal reactor; however, in the present case, $k$, $\mathrm{D}_{\mathrm{E}}$ and $\mathrm{H}$ vary with temperature. Temperature dependences of the Arrenius type for $k$ and $D_{E}$, and the reciprocal type for $H$, are taken into account in the present case.

Iterative numerical calculation is required to obtain local oxygen and active site concentrations in the fiber and oxygen concentration in the gas phase.

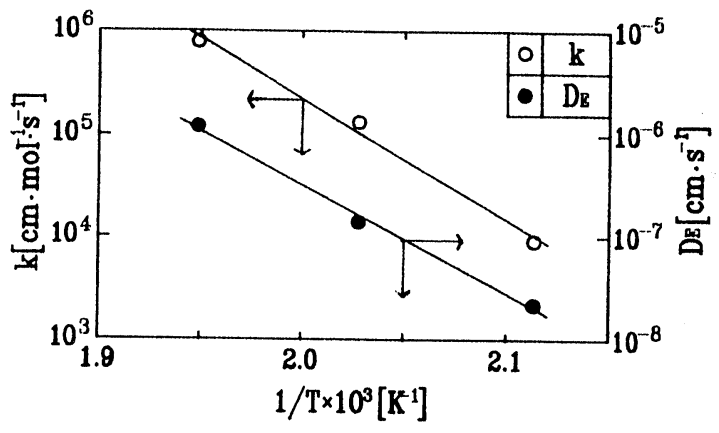

Fig. 3 Arrhenius plots of $k$ and $D_{E}$

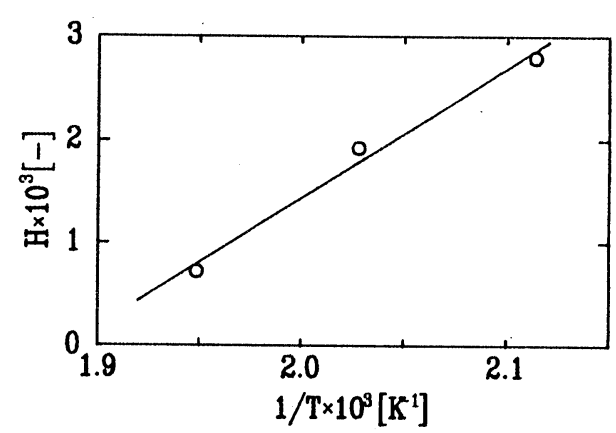

Fig. 4 Relation between $\mathrm{H}$ and $1 / \mathrm{T}$

\section{Results and Discussion}

Figure 3 illustrates the Arrhenius plot for the reaction rate constant and the effective diffusion constant given by parameter estimation calculation ${ }^{10)}$ of the isothermal kinetic model with the outlet oxygen concentrations obtained from isothermal experiments. Linear relationships are evident. The fitted straight lines were expressed as follows:

$$
\begin{aligned}
& k(T)=\left(7.36 \cdot 10^{28}\right) \exp \left(\frac{-255 \times 10^{3}}{R T}\right) \\
& D_{E}(T)=\left(4.88 \cdot 10^{14}\right) \exp \left(\frac{-203 \times 10^{3}}{R T}\right)
\end{aligned}
$$

Figure 4 shows the relationship between the gassolid equilibrium constant and the reciprocal of the temperature. The fitted straight line was expressed as follows:

$$
H(T)=\frac{126}{T}-0.237
$$

Figure 5 shows time courses of the oxygen concentrations in the outlet gas for the two heating rate conditions. Plots are experimental data and solid lines 


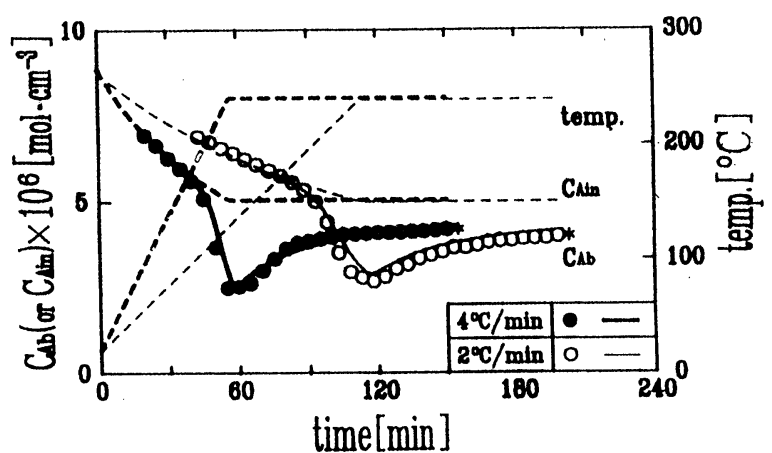

Fig. 5 Time courses of $\mathrm{O}_{2}$ concentrations and temperature in the reactor

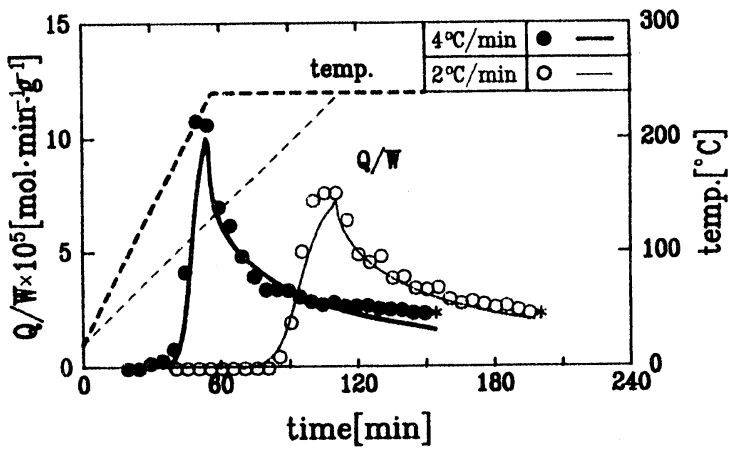

Fig. 6 Time courses of $\mathrm{O}_{2}$ consumption rate per unit mass of fiber

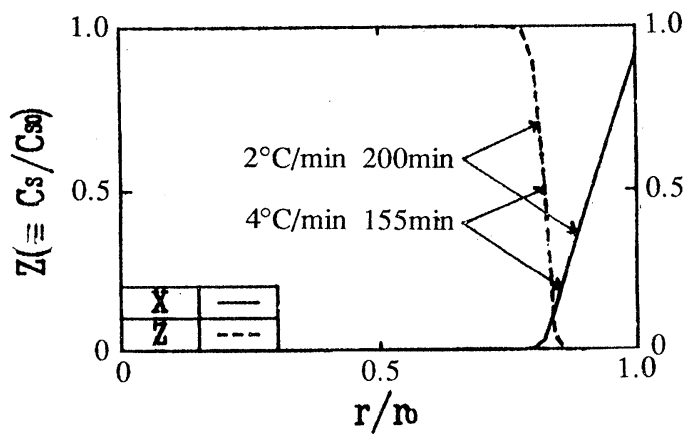

Fig. 7 Calculated distributions of non-dimensional oxygen and active site concentration at complete stabilization

represent the simulated results obtained by numerical calculation. It can be seen from the figure that a difference in oxygen concentration between the inlet and outlet of the reactor emerges at approximately $180^{\circ} \mathrm{C}$, and that the oxygen concentrations in the reactor assume minimum values when the maximum temperature is reached. It is possible to consider that the stabilization reaction begins at a temperature near $180^{\circ} \mathrm{C}$ and then accelerates with an increase in temperature. The asterisks indicate the completion of stabilization. A higher heating rate corresponds to a shorter completion time. The simulated lines for outlet concentration show excellent agreement with the plotted experimental values. This suggests that the kinetic model and numerical calculation are valid.

The rate of oxygen consumption by the fibers, Q, can be expressed by using a concentration gradient at the surface as

$$
\mathrm{Q}=2 \pi \mathrm{r}_{0} \mathrm{~L} \cdot \mathrm{D}_{\mathrm{E}} \frac{\partial \mathrm{C}_{\mathrm{A}}}{\partial \mathrm{r}} \mid \mathrm{r}=\mathrm{r}_{0}
$$

or by using a gas-phase concentration change in the reactor as

$$
\begin{gathered}
\mathrm{Q}=\left\{\mathrm{v}_{0}\left(\mathrm{C}_{\mathrm{Ain}}-\mathrm{C}_{\mathrm{Ab}}\right)-\mathrm{V} \frac{\partial \mathrm{C}_{\mathrm{Ab}}}{\partial \mathrm{t}}-\frac{\mathrm{V} \cdot \mathrm{C}_{\mathrm{AB}}}{\mathrm{T}} \cdot \frac{\partial \mathrm{T}}{\partial \mathrm{t}}\right\} / \\
\left(1 \frac{\mathrm{RT}}{\mathrm{P}} \mathrm{C}_{\mathrm{Ab}}\right)
\end{gathered}
$$

Equations (13) and (14) are utilized to obtain the theoretical and experimental values, respectively. The resulting oxygen consumption rates per unit mass of fiber, $Q / W$, are shown in Fig. 6. A higher heating rate gives a higher oxygen consumption rate when the temperature is approaching at the prescribed maximum. This is because, at that time, more active sites remain at higher heating rate condition. The agreement between experiment and theory is excellent.

Figure 7 shows the calculated distributions of nondimensional oxygen concentration $\left(X \equiv \mathrm{C}_{\mathrm{A}} / \mathrm{H} \cdot \mathrm{C}_{\mathrm{A} 0}\right)$ and active site concentration $\left(\mathrm{Z} \equiv \mathrm{C}_{\mathrm{S}} / \mathrm{C}_{\mathrm{S} 0}\right)$ in the fiber at the time when the stabilization reaction is complete. The two lines representing the respective concentrations are exactly similar. It can be observed from the figure that the oxidative stabilization is complete prior to the entire consumption of active sites in the fiber. Fixed oxygen is considered to be non-uniformly distributed in the direction of the fiber radius.

\section{Conclusions}

The oxidative stabilization of pitch fiber under increasing temperature conditions was analyzed using a mathematical model in which the temperature 
dependence of rate and equilibrium physicochemical properties was taken into account. Experimental results were well simulated by the numerical calculation of the model equations.

It is concluded that stabilization is complete, although the reaction does not proceed deeply into the fiber.

\section{References}

1) S. Otani, Carbon 3, 31 (1965).

2) S. Otani, Carbon 5, 219 (1967).

3) J-B. Donnet and R.C Bansal, "Carbon Fiber", 2nd Ed., Marcel Dekker (1990) pp. 29.

4) J.L. White and P.M. Sheaffer, Extended Abstracts of 17th Biennial Conference on Carbon, Kentucky (1985) 161.

5) E. Fitzer and D. Kompalik, Sprechsaal 119, 911
(1986).

6) W.C. Stevens and R.J. Diefendorf, Proceedings of 4th international conference, Baden-Baden (1986) 37.

7) W. Kowbel and J. Don, Extended Abstracts of 18th Biennial Conference on Carbon, Worcester (1985) 409.

8) I. Mochida, H. Toshima and Y. Korai, J. Mater. Sci. 24, 389 (1989).

9) T. Yonemoto, R. Fukushima and T. Tadaki, Tanso 1988 [No. 135] 247.

10) T. Yonemoto, T. Kawata, R. Fukushima, H. Tsutsumi and T. Tadaki, Tanso 1990 [No. 144] 177.

11) T. Yonemoto, H. Nitta, T. Kawata and T. Tadaki, Chem. Eng. J. 49, 133 (1992). 